ORIGINAL RESEARCH PAPER

\title{
THE VALIDITY OF AN X-RAY SCATTER APPROACH FOR THE DETECTION OF OLIVE OIL ADULTERATION
}

\author{
EBTESAM A. MOHAMAD ${ }^{1}$, WALAA N. ALI ${ }^{1}$, OMAR S. DESOUKY ${ }^{2}$, SEHAM M. EL- \\ MARAKBY ${ }^{2}$, WAEL M. ELSHEMEY ${ }^{1^{*}}$ \\ ${ }^{1}$ Biophysics Department, Faculty of Science, Cairo University, Giza 12613, Egypt \\ ${ }^{2}$ Department of Radiation Physics, National Center for Radiation Research and Technology, Egyptian Atomic \\ energy Authority (EAEA),Madinet Nasr 13759, Egypt \\ *Corresponding author: welshemey@sci.cu.edu.eg \\ Received on 18 February 2019 \\ Revised on 15 April 2019
}

\begin{abstract}
$\mathrm{X}$-ray scattering from biological samples was used in tissue characterization due to its dependence on the molecular structure of biological samples. This work aimed to investigate its validity in the detection of the adulteration of Virgin Olive Oil (VOO) with Corn Oil (CO) compared to the well-known viscosity and UV-Visible absorption spectroscopy techniques. Results showed that despite the favorable linear dependence of oil viscosity on the percentage of adulterant $(0 \%, 20 \%, 40 \%, 60 \%$ and $80 \%)$, yet reliable difference from pure VOO seemed to exist only for CO percentages of $40 \%$ or above. Surprisingly, X-ray scatter profile parameters - Area Under Curve (AUC) and peak height showed valid variations from pure VOO for the same CO percentages. On the other hand, UV-Visible absorption peaks at 465 and $670 \mathrm{~nm}$ showed significant differences between all concentrations of adulterated samples and control. Nevertheless, the introduction of a technique capable of probing the changes in molecular structure provides new perspective for the confirmation of the detection of VOO adulteration.
\end{abstract}

Keywords: viscosity, x-ray scattering, olive oil, corn oil, adulteration

\section{Introduction}

Food is the main source of energy for human beings therefore, it is extremely important to take care of it. Unfortunately, many types of food were subjected to adulteration by some greedy traders without any concern to human life. Adulteration of food was practiced for many decades in order to maximize the profit. Low quality ingredients were mainly used in the adulteration process instead of high quality ingredients (Nasreen and Ahmed, 2014).

Spink and Moyer (2013) pointed out that among the common adulteration methods was the replacement of some of the high quality ingredients with cheaper ones without declaring it. The adulteration process was done in a way such as consumers

https//doi.org/10.35219/foodtechnology.2019.1.10 
could not detect it. Therefore, efforts were put on offering reliable means for the detection of food adulteration (Peng et al., 2017).

Among the detected food adulterations around the globe were the adulteration of powdered milk with melamine powder (Daniel et al., 2017; Ezhilan et al., 2017; Moncayo et al., 2017). This type of adulteration was said to cause many serious health problems such as the formation of kidney stones, kidney failure and bladder cancer (Anjos et al., 2015; Wang et al., 2015; Wu et al., 2017). A widely spread beef meat adulteration with illegal meats was also discovered (Cheng et al., 2016; Nunes et al., 2016; Al-Kahtani et al., 2017; Meira et al., 2017). Butter was adulterated using margarine and palm oil (Uysal et al., 2013; Tomaszewska-Gras, 2016).

One of the important and widely spread food adulterations was the adulteration of Virgin Olive Oil (VOO) with other oils, mainly Corn Oil (CO) (Allam and Hamed, 2007; Alexa et al., 2009; Torrecilla et al., 2010; Valantina et al., 2013; Rashvand et al., 2016).

Different detection methods were used to identify food adulteration, involving Polymerase Chain Reaction (PCR) (Uysal et al., 2013), Near-Infrared (NIR) measurements (Christy et al., 2004; Huang et al., 2016), UV-Visible Spectrophotometry (Torrecilla et al., 2010; Milanez et al., 2017) and Raman Spectroscopy (Li et al., 2017). Viscosity measurements were shown to provide a means for the detection of Olive oil mixed with Corn oil (Yalcin et al., 2012; Valantina et al., 2013; Diamante and Lan, 2014; Hoffmann et al., 2018).

The present work aimed to evaluate the validity of detection of the adulteration of Virgin Olive Oil (VOO) with Corn Oil (CO) using suggested X-ray scattering technique compared to the well-known viscosity and UV-Visible absorption spectroscopy techniques. X-ray scattering has the advantage of being sensitive to the changes in the molecular structure of investigated samples and was thus expected to offer an evaluation from a completely different perspective.

\section{Materials and methods}

\section{Materials}

VOO was purchased from the Faculty of Agriculture, Cairo University, Giza, Egypt, while CO was purchased from the local market.

\section{Sample preparation}

The adulteration process was carried out through the addition of CO to VOO. The CO percentages were $20 \%, 40 \%, 60 \%$ and $80 \%$. Pure VOO was considered as control. After the addition of $\mathrm{CO}$, all blends were manually shaken for about 30 seconds and stored in dark glass bottles until measurements. These bottles were protected from light and high temperature in order to retard the oxidative process at room temperature (Allam and Hamed 2007; Alexa et al., 2009; Daniel et al., 2017).

\section{Viscosity measurements}

Viscosities of pure VOO and VOO adulterated with different percentages $(20 \%$, $40 \%, 60 \%$ and $80 \%$ ) of CO were measured. Viscosity of all samples was determined 
in triplicate. All measurements were carried out at room temperature $\left(36 \mathrm{C}^{\circ}\right)$. Brookfield DV-III, USA programmable Rheometer was used to measure the rheological properties. It is a cone-plate viscometer that measures the viscosity and the shear stress for a fluid at constant shear rate.

\section{UV-Visible absorption Spectroscopy measurements}

A UV Spectrophotometer (Jenway Ltd, Model 6405, Felsted, Dunmow, Essex, Uk, Voltage: 230/115 V, Power: 200VA, Serial No: 1125, Frequency: 50/60Hz) was used to measure the UV-Visible spectra $(200-850 \mathrm{~nm}, 5 \mathrm{~nm}$ resolution) of Virgin Olive Oil (VOO) and different percentages of the adulterant Corn Oil (CO) (Control, $20 \%, 40 \%, 60 \%$ and $80 \%)$. Quartz cuvettes $\left(1 \mathrm{~cm}^{3}\right)$ were used as sample holders. All measurements were performed in triplicate. Absorption spectra were graphed using Microsoft Excel.

\section{$X$-ray scattering measurements}

A Philip's X'pert Multipurpose X-ray Diffraction system (MPD), operating at $40 \mathrm{kV}$ and $40 \mathrm{~mA}$, with a horizontal sample holder (diameter of $25 \mathrm{~mm}$ and a depth of 5 $\mathrm{mm}$ ), was used to acquire the X-ray scattering profiles of VOO samples that were either pure or adulterated with different percentages of $\mathrm{CO}$. The device used a $\mathrm{Cu}$ target (size $12 \mathrm{~mm} \times 0.4 \mathrm{~mm}$ ) to produce $8.047 \mathrm{keV}$ collimated X-rays. All measurements were performed in step mode with a step of $0.5^{\circ}$ at an angular range from $2 \theta=4.25^{\circ}$ to $70^{\circ}$ with a step time equal to $20 \mathrm{sec}$.

\section{Results and discussion}

\section{Viscosity}

Figure 1 shows a considerably linear variation of shear stress with shear rate for almost all CO:VOO percentages. One could notice that the lower the adulterant (CO) percentage, the higher the viscosity (equal to the slope of the relation) of oil samples (Shin et al., 2010). This was further illustrated in figure 2, which shows a linear decrease in viscosity with increase in CO percentage.

Figure 2 shows the variation of viscosity with $\mathrm{CO}$ percentages added to pure VOO. The viscosity of pure VOO decreased linearly with the addition of increasing percentages of CO (Yalcin et al., 2012). One could notice that it was possible to detect the adulteration of pure $\mathrm{VOO}$ for adulterant $\mathrm{CO}$ percentages of $40 \%$ or above. Valantina et al. (2013) reported that there were many factors that may cause such decrease in viscosity including density, unsaturation and molecular weight of adulterant. López-Beceiro et al. (2011) reported that CO had linoleic acid as a dominant content in contrast to $\mathrm{VOO}$ with oleic acid as the dominant content. They attributed the increase in the VOO viscosity with the increase in the percentage of $\mathrm{CO}$ to the oleic acid/ linoleic acid ratio. Olive oil containes a lower level of saturated and higher level of non-saturated fatty acids suggesting that oils with more double bonds (e.g. CO) appear to have lower viscosity due to their loosely packed structure (Poulli et al., 2006; Sharoba and Ramadan, 2012). 


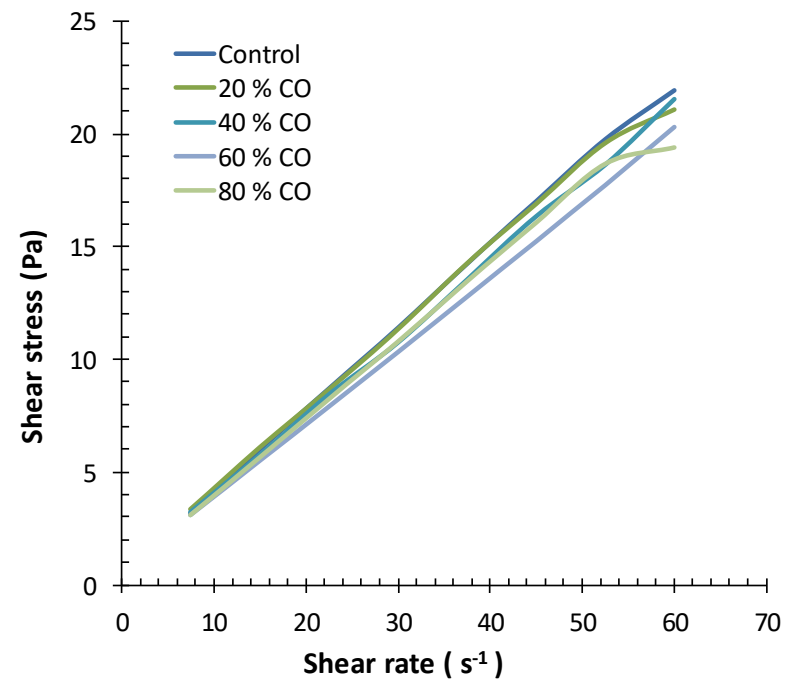

Figure 1. The variation of shear stress with shear rate for virgin olive oil adulterated with different percentages of corn oil

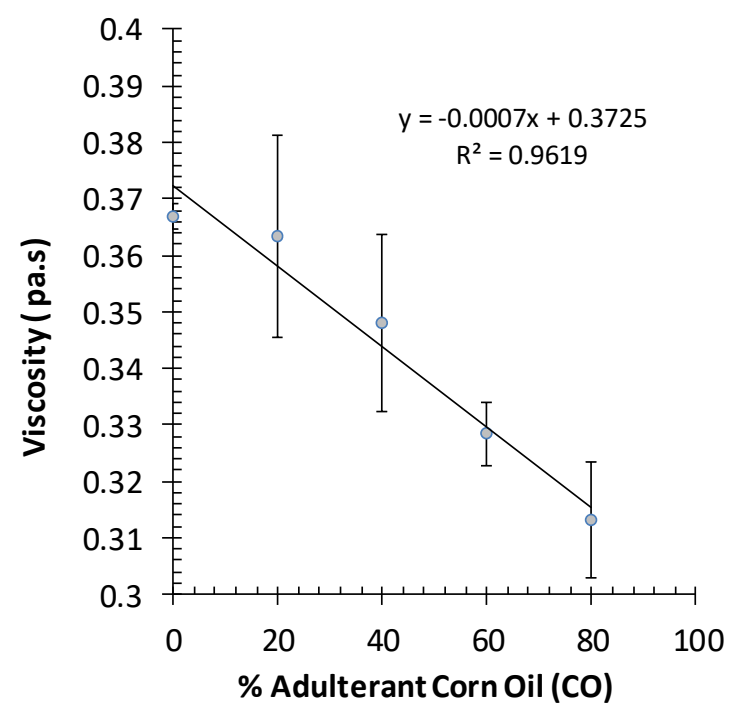

Figure 2. The variation of viscosity with percentages of adulterant corn oil in virgin olive oil samples

\section{$U V$-Visible Spectroscopy}

Figure 3a presents the UV-Visible spectra of pure VOO (Control) and Olive Oil samples adulterated with different percentages of CO $(20 \%, 40 \%, 60 \%$ and $80 \%)$. For the carotenoids absorption peak at 465 and the chlorophyll absorption peak at $670 \mathrm{~nm}$, one might notice a decrease in the value of absorbance with increase in the 
percentage of adulterant. This was better represented by the bar graph in figure $3 b$, which showed a significant $(p<0.05)$ difference between the absorbance of all adulterant concentrations and control at these two peaks. This was a favorable and expected result that was supported by the results of similar studies (Torrecilla et al., 2010; Milanez et al., 2017). The decrease in the value of absorbance with increase in the percentage of adulterant was probably due to the presence of higher percentages of chlorophyll and carotenoids in VOO compared to other seed oils (Lazzerini and Domenici, 2017).

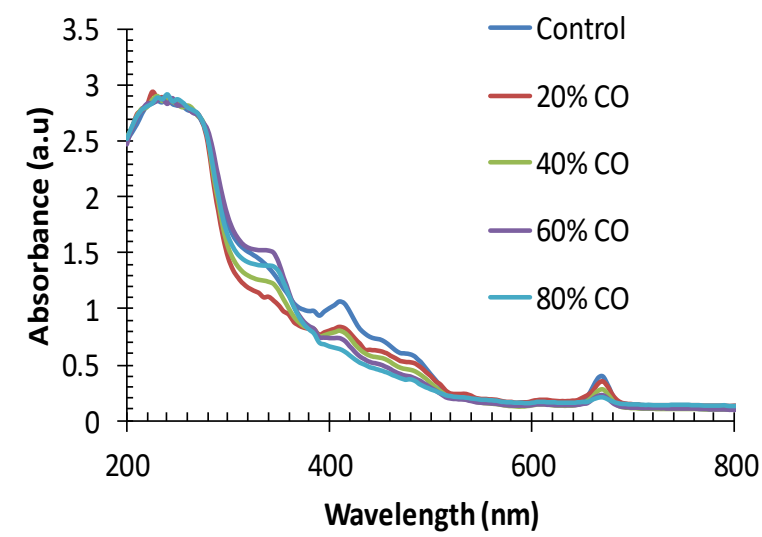

a)

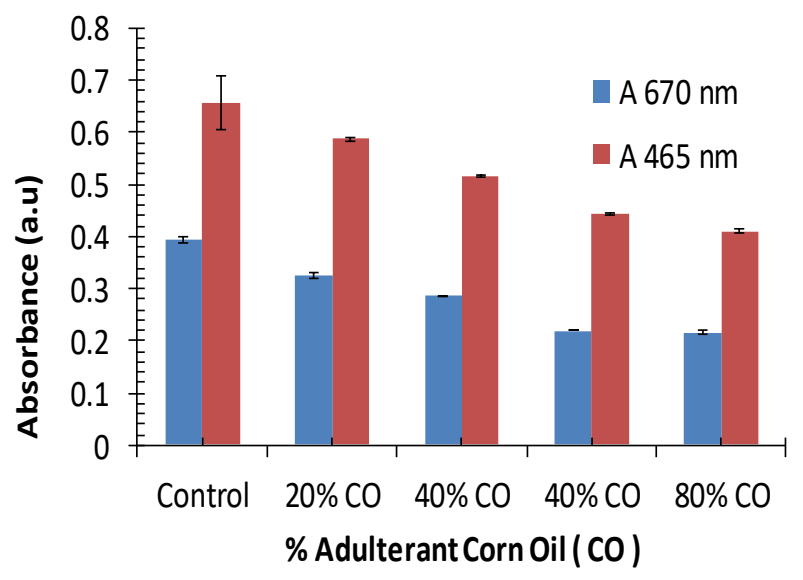

Figure 3. (a) The variation of absorbance with the wavelength, of adulterant corn oil in virgin olive oil samples (b) The variation of absorbance with different adulterant percentages at 670 $\mathrm{nm}$ and $465 \mathrm{~nm}$.

\section{$X$-ray scattering}

Figure 4 presents normalized (at theta $=41.25^{\circ}$ ) X-ray scattering profiles of pure VOO and oil samples containing different percentages of adulterant $\mathrm{CO}$ added to 
VOO. One could notice that it is possible to differentiate the profiles containing CO percentages of $40 \%$ or above from pure VOO. Figure 5 shows the variation of Area Under Curve (AUC) and Peak height X-ray scatter parameters with adulterant CO percentages in VOO samples. Both parameters could differentiate $\mathrm{CO}$ percentages of $40 \%$ or above from pure VOO, where the Peak height X-ray scatter parameter showed slightly larger differences between adulterated samples and the pure VOO sample.

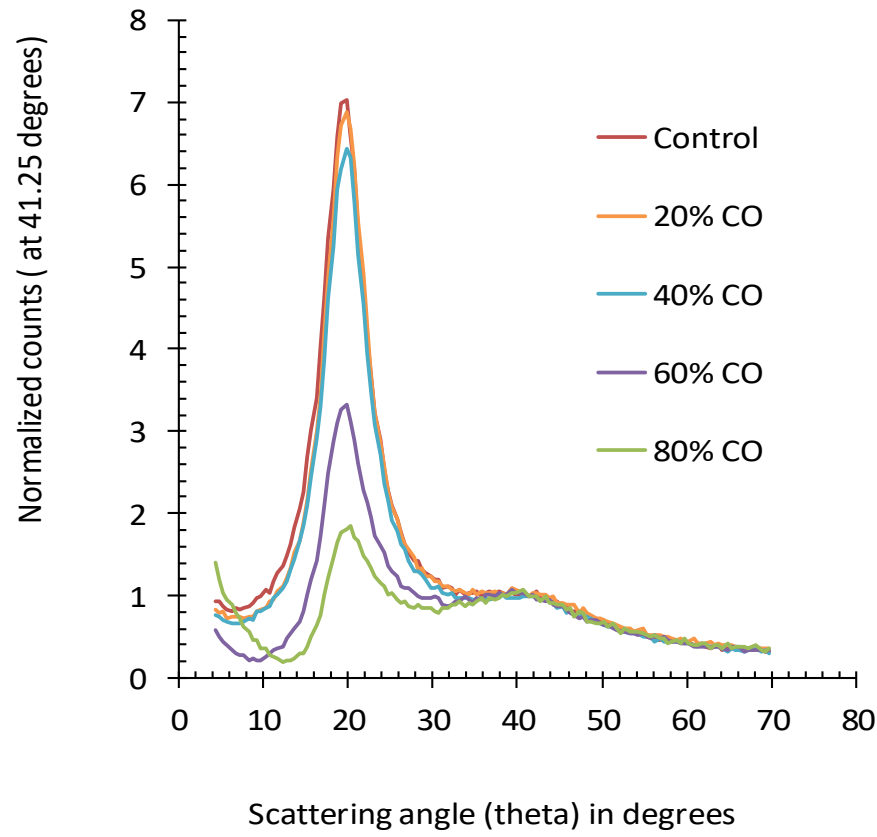

Figure 4. Peak normalized (at theta $=41.25^{\circ}$ ) X-ray scattering profiles for pure virgin olive oil and different percentages of corn oil added as adulterant to virgin olive oil.

The X-ray scattering results were compatible with those obtained from viscosity measurements. This was a success for the X-ray scattering technique that was investigated for such purpose for the first time. The technique is unique in that it depends on the detection of changes in molecular structure of given samples (Elshemey et al., 2013; Elsharkawy and Elshemey, 2013; Elshemey et al., 2015). Nevertheless, the results obtained by both techniques, although promising would probably need enhancements for the detection of adulterant CO below $40 \%$. 


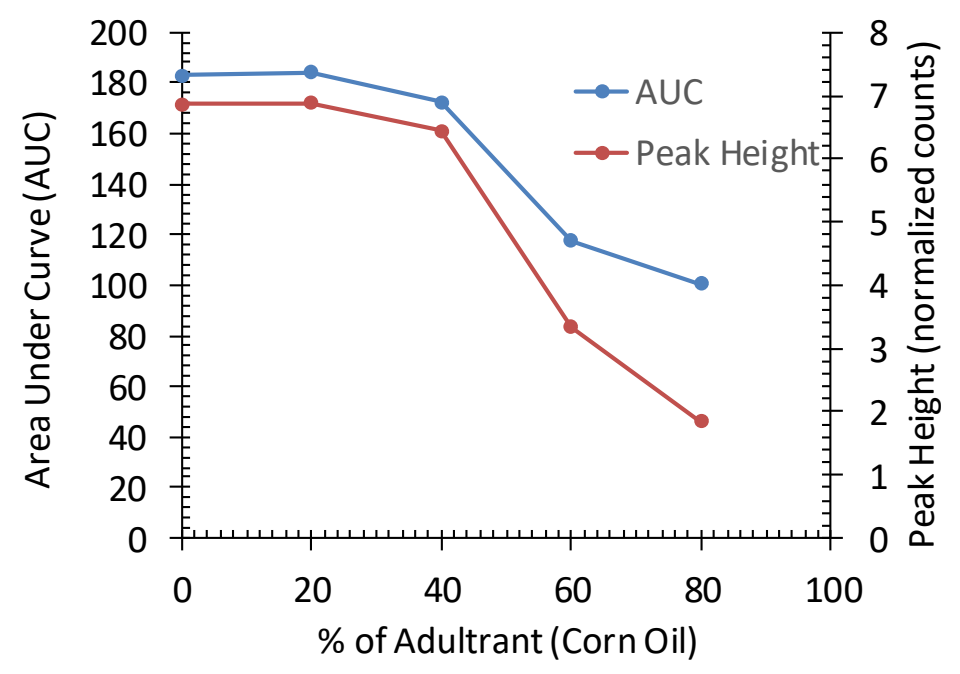

Figure 5. The variation of Area Under Curve (AUC) and Peak Height X-ray scatter parameters with adulterant corn oil percentages in virgin olive oil samples.

\section{Conclusions}

X-ray scattering technique showed a performance compatible to viscosity measurements in the detection of adulteration of $\mathrm{VOO}$ with different percentages of CO. Although limited by a minimum $\mathrm{CO}$ of $40 \%$, further investigations for the improvement of this technique might enhance its limit for detection of adulterant $\mathrm{CO}$ in VOO samples. Despite UV-Visible spectroscopy showed better performance, yet the merit in using x-ray technique being its difference from the currently available techniques in that it depended on changes in the molecular structure of investigated samples and consequently, it offered a complementary verification from completely different perspective.

\section{References}

Alexa, E., Dragomirescu, A., Pop, G., Jianu, C., Dragos, D. 2009. The use of FT-IR spectroscopy in the identification of vegetable oils adulteration. Journal of Food Agriculture \& Environment, 7, 20-24.

Al-Kahtani, H.A., Ismail, E.A., Ahmed, M.A. 2017. Pork detection in binary meat mixtures and some commercial food products using conventional and real-time PCR techniques. Food Chemistry, 219, 54-60.

Allam, M.A., Hamed, S.F. 2007. Application of FTIR spectroscopy in the assessment of olive oil adulteration. Journal of Applied Sciences Research, 3, 102-108.

Anjos, O., Campos, M.G., Ruiz, P.C. Antunes, P. 2015. Application of FTIR-ATR spectroscopy to the quantification of sugar in honey. Food Chemistry, 169, 218-223.

Cheng, J.H., Chou, H.T., Lee, M.S., Sheu, S.C. 2016. Development of qualitative and quantitative PCR analysis for meat adulteration from RNA samples. Food Chemistry, 192, 336-342. 
Christy, A.A., Kasemsumran, S., Du, Y., Ozaki, Y. 2004. The detection and quantification of adulteration in olive oil by near-infrared spectroscopy and chemometrics. Analytical Sciences, 20, 935-940.

Daniel, S.K., Julius, L.A.N., Gorthi, S.S. 2017. Instantaneous detection of melamine by interference biosynthesis of silver nanoparticles. Sensors and Actuators B: Chemical, 238, 641-650.

Diamante, L.M., Lan, T. 2014. Absolute viscosities of vegetable oils at different temperatures and shear rate range of 64.5 to $4835 \mathrm{~s}^{-1}$. Journal of Food Processing. ID 234583, 6p.

Elshemey, W.M., Mohamed, F.S., Khater, I.M. 2013. X-ray scattering for the characterization of lyophilized breast tissue samples. Radiation Physics and Chemistry, 90, 67-72.

Elsharkawy, W.B., Elshemey, W.M. 2013. Quantitative characterization of fatty liver disease using x-ray scattering. Radiation Physics and Chemistry, 92, 14-21.

Elshemey, W.M, Rady, A.S., Dakrory, A. 2015. X-ray scattering: A structure based method for the assessment of fat quality and fat content in beef products. Food Biophysics, 10, $76-82$.

Ezhilan, M., Gumpu, M.B., Ramachandra, B.L., Nesakumar, N., Babu K.J., Krishnan, U.M., Rayappan, J.B.B. 2017. Design and development of electrochemical biosensor for the simultaneous detection of melamine and urea in adulterated milk samples. Sensors and Actuators B: Chemical, 238, 1283-1292.

Hoffmann, J.F., Vaitilingom, G., Henry, J.F., Chirtoc, M., Olives, R., Goetz, V., Py, X. 2018. Temperature dependence of thermophysical and rheological properties of seven vegetable oils in view of their use as heat transfer fluids in concentrated solar plants. Solar Energy Materials and Solar Cells, 178, 129-138.

Huang, M., Kim, M.S., Delwiche, S.R., Chao, K., Qin, J., Mo, C., Esquerre, C., Zhu, Q. 2016. Quantitative analysis of melamine in milk powders using near-infrared hyperspectral imaging and band ratio. Journal of Food Engineering, 181, 10-19.

Lazzerini, C., Domenici, V., 2017. Pigments in extra-virgin olive oils produced in Tuscany (Italy) in different years. Foods, 6(4), 25

Li, R., Yang, J., Han, J., Liu, J., Huang M. 2017. Quantitative determination of melamine in milk using Ag nanoparticle monolayer film as SERS substrate. Physica E: Lowdimensional Systems and Nanostructures, 88, 164-168.

López-Beceiro, J., Artiaga, R., Gracia, C., Tarrío-Saavedra, J., Naya, S., Mier. J.L. 2011. Comparison of olive, corn, soybean and sunflower oils by PDSC. Journal of Thermal Analysis and Calorimetry, 104, 169-175.

Martín, I.G., Macias, E.M., Sánchez, J.S., Rivera, B.G. 1998. Detection of honey adulteration with beet sugar using stable isotope methodology. Food Chemistry, 61(3), 281-286.

Meira L., Costa J., Villa C., Ramos F., Oliveira M. B., Mafra I. 2017. EvaGreen real-time PCR to determine horse meat adulteration in processed foods. LWT-Food Science and Technology, 75, 408-416.

Milanez K.D., Nóbrega T.C.A., Nascimento D.S., Insausti M., Band B.S.F., Pontes M.J.C. 2017. Multivariate modeling for detecting adulteration of extra virgin olive oil with soybean oil using fluorescence and UV-Vis spectroscopies: A preliminary approach. LWT-Food Science and Technology, 85, 9-15.

Moncayo S., Manzoor S., Rosales J.D., Anzano J., Caceres J.O. 2017. Qualitative and quantitative analysis of milk for the detection of adulteration by Laser Induced Breakdown Spectroscopy (LIBS). Food Chemistry, 232, 322-328.

Nasreen S., Ahmed T. 2014. Food adulteration and consumer awareness in Dhaka City 19952011. Journal of Health, Population and Nutrition, 32, 452-464. 
Nunes K.M., Andrade M.V., Santos Filho A.M., Lasmar M.C., Sena M.M. 2016. Detection and characterisation of frauds in bovine meat in natura by non-meat ingredient additions using data fusion of chemical parameters and ATR-FTIR spectroscopy. Food Chemistry, 205, 14-22.

Peng G.J., Chang M.H., Fang M., Liao C.D., Tsai C.F., Tseng S.H., Kao Y., Chou H., Cheng H.F. 2017. Incidents of major food adulteration in Taiwan between 2011 and 2015. Food Control, 72, 145-152.

Poulli K.I., Mousdis G.A., Georgiou C.A. 2006. Synchronous fluorescence spectroscopy for quantitative determination of virgin olive oil adulteration with sunflower oil. Analytical and Bioanalytical Chemistry, 386, 1571-1575.

Rashvand M., Omid M., Mobli H., Firouz M.S. 2016. Adulteration detection in olive oil using dielectric technique and data mining. Sensing and Bio-Sensing Research, 11, 33-36.

Sharoba A.M., Ramadan M.F. 2012. Impact of frying on fatty acid profile and rheological behaviour of some vegetable oils. Journal of Food Processing and Technology, 3, 161.

Spink J., Moyer D.C. 2013. Understanding and combating food fraud. Food Technology, 67, 30-35.

Tomaszewska-Gras J. 2016. Rapid quantitative determination of butter adulteration with palm oil using the DSC technique. Food Control, 60, 629-635.

Torrecilla J.S., Rojo E., Dominguez J.C., Rodríguez F. 2010. A novel method to quantify the adulteration of extra virgin olive oil with low-grade olive oils by UV-Vis. Journal of Agricultural and Food Chemistry, 58, 1679-1684.

Uysal R.S., Boyaci I.H., Genis H.E., Tamer U. 2013. Determination of butter adulteration with margarine using Raman spectroscopy. Food chemistry, 141, 4397-4403.

Valantina S.R., Chandiramouli R., Neelamegam P. 2013. Detection of adulteration in olive oil using rheological and ultrasonic parameters. International Food Research Journal, 20, 3197-3202.

Wang S., Guo Q., Wang L., Lin L., Shi H., Cao H., Cao B. 2015. Detection of honey adulteration with starch syrup by high performance liquid chromatography. Food Chemistry, 172, 669-674.

Wu L., Du B., Vander Heyden Y., Chen L., Zhao L., Wang M., Xue X. 2017. Recent advancements in detecting sugar-based adulterants in honey-A challenge. Trends in Analytical Chemistry, 86, 25-38.

Yalcin H., Toker O.S., Ozturk I., Dogan M., Kisi, O. 2012. Prediction of fatty acid composition of vegetable oils based on rheological measurements using nonlinear models. European Journal of Lipid Science and Technology, 114, 1217-1224.

Zhang W., Xue J. (2016): Economically motivated food fraud and adulteration in China: An analysis based on 1553 media reports. Food Control, 67, 192-198. 